\title{
ASSESSMENT OF SUSTAINABLE NEIGHBOURHOODS: FROM STANDARDS TO CULTURAL PRACTICES
}

\author{
C. DOUSSARD \\ Paris 1 Panthéon Sorbonne University, Paris, France.
}

\begin{abstract}
The development of Neighbourhood Sustainability Assessment (NSA) tools has contributed to the planning and design of better urban environments. Some of these systems aim to be universal through the use of standards, while paradoxically contributing to the certification of projects in culturally diverse contexts. This practice, however, is inconsistent with the notion of sustainability that advocates for a planning and design process adapted to a specific location. Therefore, this study examines how some national NSA tools adapt or not to other countries' cultures while using standards as a mean of evaluation. It provides an analysis of four NSA systems, the LEED-ND v2009 rating tool, the HQE-A label, the AQUA Bairro e loteamento label, and HKTS green architecture award, conceived by American, French, Brazilian and Vietnamese organisations, respectively. It examines through the survey and classification of evaluation parameters to which extent an assessment culture is sometime contradictory to the assessment of neighbourhoods developed in other cultural contexts. The study ultimately leads to the characterisation of distinct NSA tools cultural profiles. Finally, it discusses the factors (such as contextual adaptation, planning and design ideology, and green construction market growth) that drive the conception of NSA tool.

Keywords: assessment culture, eco-neighbourhood certification, standards, sustainable neighbourhood assessment system, urban sustainability.
\end{abstract}

\section{INTRODUCTION}

Since the 2000s, Neighbourhood Sustainability Assessment (NSA)tools have increasingly contributed to the certification of projects worldwide [1]. This trend responds to the need of establishing a common framework for future community planning and design, following the release of the 1987 Brundtland report defining the concept of "sustainability" [2, 3]. The present study considers an NSA system as a specific type of impact assessment, which estimates the difference between what would happen if a neighbourhood were built sustainably in comparison to if it were not. NSA tools can also constitute rating systems and are often associated with the delivery of certificates, labels or awards. They can either be applied regionally, or on the contrary, aim to be universal. In the latter case, NSA tools are mainly based on standards, which is contradictory to sustainability principles advocating for a design and planning adapted to a specific local context and culture [4]. So how do these assessment systems that inform the planning and design processes of sustainable neighbourhoods get adapted to a specific context while responding to international norms? What are the evaluation parameters that inform a given NSA culture? And is such an assessment culture consequently adapted to a specific local context?

This article addresses these questions first through the historical and cultural explorations of what constitutes the assessment of sustainable neighbourhoods. Second, it presents the 
analysis of four certification systems originating from France, the United States, Brazil and Vietnam. It is based on a systematic survey and characterisation of assessment parameters used by those rating tools. Even though many comparisons of certification systems exist in literature [5-8], it seems that no previous work concentrates on what constitute their cultural specificities. Finally, the article discusses which factors drive the establishment of such characteristics, and therefore explains their relative influence on the design of NSA tools at a global scale.

\section{HISTORICAL, CULTURAL AND METHODOLOGICAL PERSPECTIVES}

\subsection{Historical developments}

The assessment of urban environment is not a recent process. After the 1950s, urban planning practices shifted from the paradigm associated with Patrick Geddes's survey-analysis-plan to rational planning. Ever since, evaluation was considered as a part of the planning process, leading to the design of many impact assessment tools [9]. The concept of impact assessment relates to a very common form of evaluation used in the context of urban planning, and is largely the product of the last fifty years. In the late 1960s, several environmental crises emerged, especially in the United States, as unrestrained economic development following World War II raised concerns among scholars [10]. However, most industrialized nations addressed these crises, and in 1969, the United States was the first country to enact environmental impact assessments as a mandatory practice for evaluating planning projects. Further developments involved the creation of The International Association for Impact Assessment in 1981 to facilitate communication between scholars involved in the theory and practice of such assessments. The development of similar organisations around the world contributed to the release of several practical evaluation systems over the years [11].

However, the establishment of sustainability assessment tools contributed to a major shift in the field of impact assessments. This new movement rose during the early 1990s, following the global recognition of the sustainable development concept. Sustainability assessment systems then encouraged the planning and design of more socially equal, economically viable and environmentally friendly communities [3]. However, this movement focused first on the building scale. It started when BREEAM, launched in 1990 in the United Kingdom, began to provide guidelines and a set of standards to the construction industry for the design of sustainable buildings [12]. The expansion and multiplication of building assessment tools around the world preceded another movement, which leaned towards the evaluation of the intermediate level of neighbourhood. This trend, which began a decade ago, can be explained by two main factors. First, the neighbourhood unit is the minimum scale to take the social, economic and institutional dimensions of sustainability into account. Second, even though the use of NSA tools is a more challenging task, it does not face the problem of socio-spatial heterogeneity that the municipal and metropolitan scales present [13].

\subsection{Standards versus culture: perspectives}

Consequently, many NSA tools were conceived over the last 10 years, and some of the organisations that designed those tools exported them to develop their activities internationally. LEED is for example the world market leader, as it certified 143 neighbourhoods in 
7 countries as of 2015 [14]. To successfully export their assessment model, organisations such as LEED designed those tools based on standards. A standard is considered here as a document that "provides requirements, specifications, guidelines or characteristics that can be used consistently to ensure that materials, products, processes and services are fit for their purpose" [15]. However, the exportation of such NSA tools is paradoxical, as sustainability principles advocate for local design and planning strategies that cannot be "used consistently" all the time.

Moreover, sustainable urban practices are comprehended and assessed through culturally specific forms of knowledge, a dimension that remains under-researched today. To successfully design and assess neighbourhoods, it is necessary to understand the cultural reasons that lead to unsustainable urban development. Therefore, NSA tools are here addressed and discussed as cultural expressions, as they were designed by different sets of stakeholders from different geographical locations. These stakeholders apprehend space and the practice of sustainable assessment through the lens of the culture in which they have been professionally active. However, they might neglect the importance of their own cultural viewpoint when designing an evaluation model susceptible to being adapted to various contexts. Indeed, organisations such as LEED and BREEAM that export those systems claim to consider all local situations, while still using normative tools to rate projects [4]. Therefore, to achieve an assessment adapted to any regional context, those NSA tools present a mixed structure both based on the use of international standards and on more local and culturally specific evaluation parameters.

\subsection{Methodological approach}

This article explores the structures of several NSA tools through the lens of both standardized and culturally specific evaluation parameters. We confronted four different certification systems: the American LEED-ND v2009 rating system, the French HQE-A rating tool, the Brazilian AQUA-Bairros e loteamento assessment system, and the Vietnamese HKTS Green Project Award.

These NSA tools were chosen for the following reasons:

- They were all designed and implemented after 2005 by organisations located on four continents, therefore maximising the tools' cultural differences;

- They either tend to be exported and used as they are abroad (LEED-ND), or are the product of another NSA tools local adaptation (AQUA-Bairros e loteamento based on the HQE-A rating tool), or present no official lineage with other NSA tools and do not pretend to be exported (HKTS Green Project Award).

The study evaluates the structure of each NSA tools through the survey, characterisation and classification of each parameter involved in the assessment of projects.

As all NSA systems consider sustainability as a driver to their certification approach, the first step of the research entailed the classification of their criteria through an assessment grid taking into consideration environmental, social and economic aspects. As environmental parameters were overrepresented, we split the related dimension in two sub-categories: resources and livability. We also established a fifth category that included criteria that could not be classified according to sustainability principles (for instance the degree of project completion or the hiring of a LEED professional). 
Second, we also analysed the nature of each indicator according to the following categories: the evaluation criterion constitutes (1) a standard, (2) is a standard, but includes a contextualized component (e.g. assessment parameters such as "the local population must be included in the design process"), (3) does not respond to the previous (1) and (2) definitions.

Third, we classified the characterized assessment parameters in relation to their degree of cultural specificity. These therefore are (1) not culturally specific, as all NSA tools include that criterion in their assessment model, (2) presenting degrees of cultural similarities, as two or three out of the four studied NSA systems share this parameter, (3) cultural singularities, as only one NSA tool uses this indicator to assess projects.

Finally, we calculated the percentages representing the number of points allocated to each category when it was statistically relevant.

\section{NSA TOOLS CHARACTERISTICS AND TYPES}

\subsection{Results from the survey}

Using the above methodology, our study identified several NSA tools profiles when comparing common, partially shared, and culturally specific criteria.

First, we identified 10 non-culturally specific parameters common to all NSA systems. These, while representing on average $23 \%$ of the criteria used for each assessment tool, only constitute $9.5 \%$ of all surveyed parameters. Moreover, the findings demonstrate that most of these common parameters relate to the two environmental sub-categories, which is consistent with previous results presented in literature. These environmental parameters are also the most standardized, especially in the case of the LEED-ND and AQUA rating tools. On the other hand, only three social and economical assessment parameters are common to all rating systems. This result is logical as social and economic parameters only represent $17 \%$ and $8 \%$, respectively, of all considered criteria, while also being less standardized. See Table 1 for more details.

The second analytical category relates to assessment criteria that are used by two or three NSA tools but not the four of them. As demonstrated in Fig. 1, most of the rating tools present between $29 \%$ and $39.6 \%$ of partially shared criteria, once again mainly environmental. However, there is an exception to this observation, as both AQUA and HQE-A profiles exceed this range. Indeed, most of their evaluation parameters are similar, even though the HQE-A system presents two criteria that the AQUA tool does not consider. These are the "area of solar panels used per project" and the "proportion of green spaces associated with ecological corridors". The lineage between both systems is here very clear in terms of parameter selection. However, even though they use the same framework, they do not adopt the same proportion of standards to assess projects. Indeed, while AQUA evaluations are mainly based on norms, it is the opposite for the HQE-A rating tool. When it comes to the proportion of standards used, the LEED-ND and AQUA systems present more standardized parameters than the HQE-A and HKTS tools. Furthermore, when looking in detail, environmental parameters are more normalized than the ones classified in the socio-economical and "Other" categories. The analysis of the standards two sub-categories repartition reveals that those including a contextual component are mainly used to assess parameters related to livability. On the other hand, standards that do not are better considered for evaluating the use of environmental resources. This examination supports observations made earlier for non-culturally specific criteria. 
16 Urban Regeneration and Sustainability

Table 1: NSA tool common assessment parameters characterisation.

\begin{tabular}{|c|c|c|c|c|c|}
\hline & & LEED-ND & HQE-A & AQUA & HKTS \\
\hline $\begin{array}{l}\text { Environment } \\
\text { resources }\end{array}$ & $\begin{array}{l}\text { Energy efficiency } \\
\text { Water management } \\
\text { Waste infrastructure } \\
\text { Biodiversity preservastion }\end{array}$ & $\begin{array}{l}\bullet \\
\bullet \\
\circ\end{array}$ & $\bullet$ & • & $\bullet$ \\
\hline Environment & Density & $\bullet$ & & $\bullet$ & \\
\hline livability & $\begin{array}{l}\text { Access to public transportation } \\
\text { Architectural quality }\end{array}$ & ० & & o & \\
\hline Social & $\begin{array}{l}\text { Heritage preservation } \\
\text { Social mixity }\end{array}$ & $\bullet$ & & $\bullet$ & \\
\hline Economic & Mixed-use environment & $\bullet$ & & & \\
\hline
\end{tabular}

- Standard

- Standard with a contextualized component

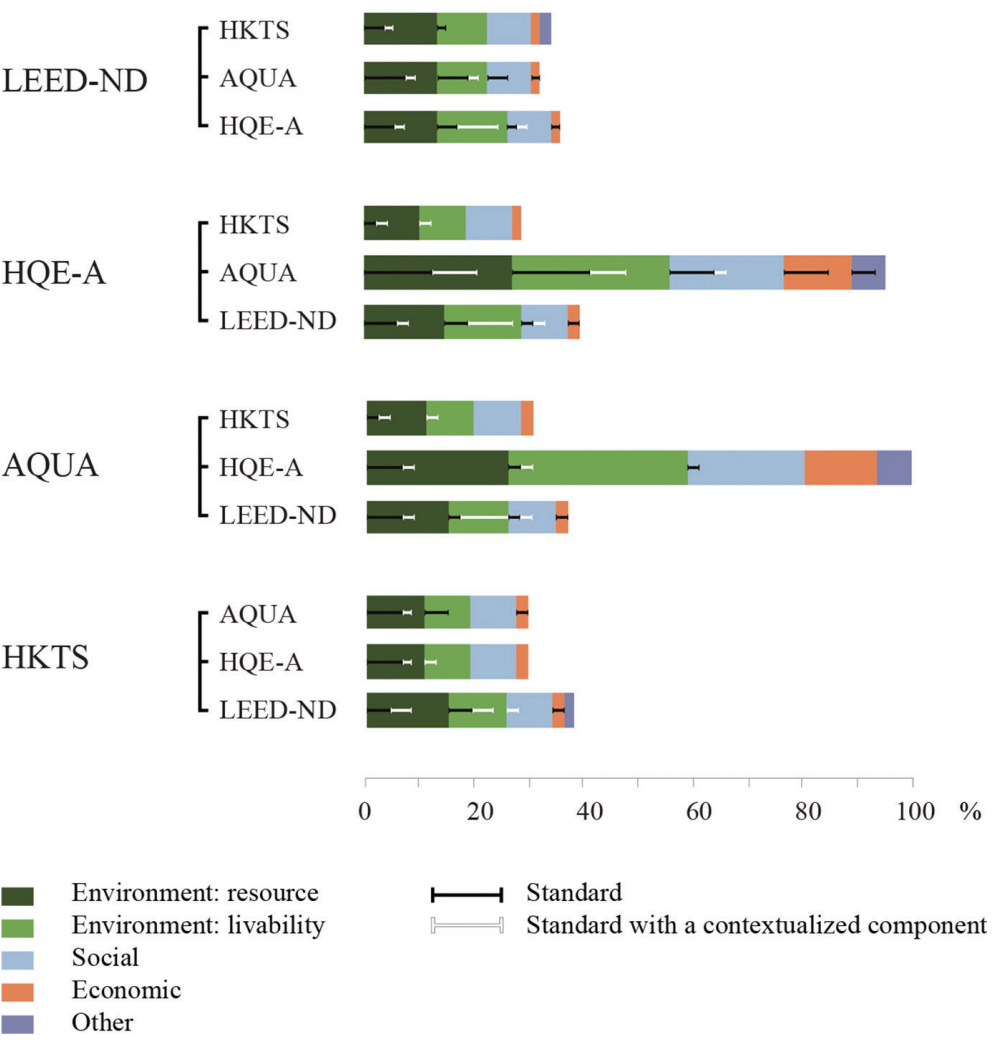

Figure 1: Characterisation of NSAT degrees of shared assessment parameters. 


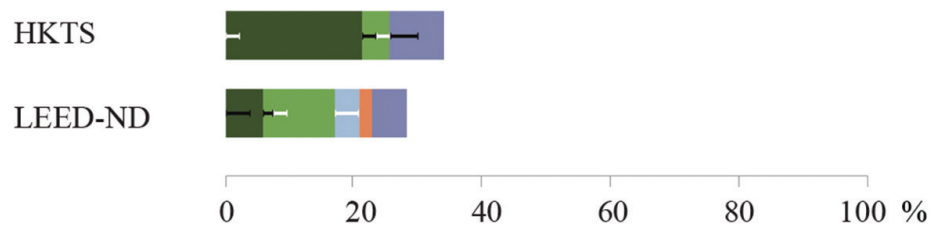

\begin{tabular}{|l} 
Environment: resource \\
Environment: livability \\
Social \\
Economic \\
Other
\end{tabular}

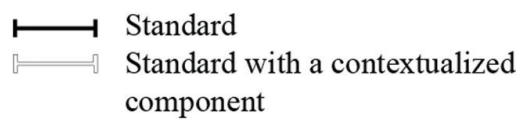

Figure 2: Characterisation of NSAT culturally specific parameters.

The investigation of the last category, related to criteria that represent assessment culture singularities, finalizes the results exposed above. The outcome of our survey is presented in Fig. 2. As the HQE-A and AQUA systems derive from one another, no culturally specific parameters are observed here. However, this is not the case for LEED-ND and HKTS that respectively present $24.5 \%$ and $35 \%$ of such parameters. Most of the considered indicators are then environmental and classified in the "Other" category. However, when looking more closely at those criteria, a paradox emerges. Indeed, even though those are only used by one NSA tools and consequently categorized as culturally specific, those parameters are not necessarily relevant to the tool's geographical origin. This fact is clear when looking at the LEED-ND parameters, two of which are "visitability and universal design", as well as "regional priority credits". In the first case, the notion of "universal design" contradicts the notion that a culturally specific criterion is inevitably relevant to a given local context. Such a statement is also supported by the case of the "regional priority credits", which requires a selection of a flexible set of criteria that can be adapted to several regional contexts. However, this observation is not always true, as an indicator adopted by a given assessment culture can also be completely relevant to its related cultural context. For instance, the HKTS system does present culturally specific criteria that are adapted to the Vietnamese context, especially in the field of intangible urban practices and social relationships.

\subsection{NSA tools profiles}

The analysis of the parameter characterisation sheds light on the several strategies NSA tools use to adapt to a given cultural context. Therefore, this study led to the establishment of the following profile types:

- MSCS: Mostly Standardized, Culturally Specific (LEED-ND);

- MSNC: Mostly Standardized, Not Culturally specific (AQUA);

- RSCS: Rarely Standardized, Culturally Specific (HKTS);

- RSNC: Rarely Standardized, Not Culturally specific (HQE-A).

NSA systems that present an MSCS profile are similar to the LEED-ND rating tool. LEED's strategy to export their model is based on the use of standards that can be contextual- 
ized. This tool, designed through the lens of the American assessment culture, is used as it is, whether a project is assessed in the United States or abroad. However, as expressed before, the fact such tools are culturally specific does not necessarily imply that the parameters involved to assess projects make it particular to a specific geographical region. The "regional priority credit", a flexible pool of assessment parameters chosen by the project managers, is then the main factor that contributes to the adaptation of the model to other local contexts and cultures.

MSNC profiles, represented here by the AQUA model also adopted a majority of standardized parameters. However, contrary to the one chosen by LEED, these are specifically adapted to the Brazilian context, and consequently constitute an NSA tool that is not meant to be exported. Moreover, these profiles do not necessarily present a specific culture of assessment, as they are the by-products of another country's assessment tool.

Organisations such as HQE, which also tend to export their assessment system, use a different strategy involving NSA tools presenting a RSNC profile. They are not culturally specific when it comes to the selection of parameters involved in the assessment. However, those parameters are mainly not standardized, which allows project managers abroad to apply their own country's norms to the system criteria, as demonstrated through the AQUA case. In the nation where this model is designed, such as France for HQE-A, the NSA tool has to comply with the country's norms. However, the project managers have to define themselves higher performance objectives to get certified.

Finally systems presenting RSCS profiles such as the HKTS award operate another assessment strategy. The goal of the organisation that designs them is to only assess national projects which, while complying with the country's norms, have to perform better in terms of sustainability objectives.

\section{DRIVING FACTORS}

This last section concentrates on the significance of NSA tool international developments and framework transfers. How can we understand these trends and demands for neighbourhood assessment? Answers can be found in the combination of the following three factors that apparently drive such a phenomenon: contextual adaptations, urban design and planning ideology, and marketing strategies.

\subsection{Contextual adaptation}

Because NSA systems by definition anticipate what would happen if a neighbourhood were built sustainably, they consequently consider the (non sustainable) context in which the project is built. As a sustainable project is defined through its environmental, social and economic dimensions, experts need to consider those aspects when designing a NSA tool for a given context.

As we saw, not only do all studied NSA systems put greater emphasis on environmental parameters, but those criteria are also the ones that present the most similarities. Energy efficiency, water management, biodiversity preservation and density are some of those equally considered by each assessment culture. However, the way they are evaluated depends on the geographical context in which they are applied. For instance, Brazil is one of the world's main biodiversity hotspots, and cities such as Rio de Janeiro present high proportions of preserved natural areas [16]. Consequently, the AQUA tool did not consider the criterion "proportion of green spaces associated with ecological corridors" included in the HQE-A 
system, as it is not as relevant to evaluate as it is in French more mineral urban environments. Another example: the AQUA tool does not present the same normative thresholds as LEEDND in terms of heating and energy consumption. The tropical climate of Brazil is indeed not the same as the climate of the United States' North East for instance. Energy consumption not only depends on climatic and geographical conditions, but it is also linked to socio-economical pressures. As developed countries consume and produce more in terms of GDP, they rely more on devices and urban processes that require more energy than population from developing countries [17]. Furthermore, socio-economical variables can be more complex to evaluate, and sometimes be the source of politically oriented debates when it comes to their interpretation. Consequently, they are less considered by NSA tools, which concentrate more on parameters that relates to the scientific measurement of a given environmental urban condition.

\subsection{Urban planning and design ideology}

Planning ideology and education also play a role when coming to the different NSA tools design and deployment strategies analysed earlier.

As developed and western countries were historically the first to design impact assessments tools, they are still today orienting the discourse about NSA system design [12]. Out of the 21 most well-known and cited NSA tools in literature, 16 were designed in developed countries, 12 of which are considered as western [5]. NSA systems though developed through the lens of the globally adopted concept of sustainability, are consequently the product of a certain sustainable planning and design ideology coming from developed, Western countries. One explanation could be that those nations have at their disposal the capital, as well as present a more favourable environment for building costly projects than conventional neighbourhoods. However, this article argues that education could play a subtler role.

Indeed, the theorisation of sustainability at the scale of the neighbourhood is the result of various movements established by Western scholars since the early 20th century [10]. These experts historically contributed to develop NSA tools, while also spreading knowledge about sustainable neighbourhood design and planning through two main channels. First, by teaching in design, planning and engineering schools, sharing theoretical insights and techniques susceptible to be deployed by future practitioners. Second, through the intervention of the same organisations that designed NSA tools. For instance, the USGBC provides LEED credentials to professionals willing to be involved in the assessment of sustainable projects. It is today the market leader, as 200,500 LEED professionals contribute to the certification of sustainable constructions globally. These professionals are however mostly accredited for projects developed at the building scale. When it comes to neighbourhoods, 211 professionals, mainly architects engineers and urban designers practicing in 21 countries, got their LEED-ND accreditation as of 2016 [18]. Consequently, these practitioners contribute to spread the LEED assessment culture, while working in other cultural contexts.

\subsection{Green construction market growth}

The development of NSA tools responds to the growth of the sustainable construction market worldwide. Few scholarly studies, however, concentrate on the subject, and analyse trends at the architectural scale more than at the scale of the neighbourhood. Moreover, they mainly focus on the environmental aspect of sustainable construction. The World Green Building 
Trends 2016 report, one of the most comprehensive surveys of the global green construction markets to date [19], focuses on this topic. It presents the analysis of the global green construction market evolution, based on the results from a survey sent to respondents in 69 countries. Today, $17 \%$ of the construction firms from these nations certify more than $60 \%$ of their projects by organisations such as LEED. By 2018, it is estimated that this percentage will double and reach $33 \%$. However, countries with developing markets such as Asia, the MENA region (Middle East/North Africa), Sub-Saharan Africa and South America are the main drivers of this anticipated growth. These are also the regions that do not necessarily dispose of their own NSA tools and are susceptible to using existing tools. Such regions could constitute markets providing new opportunities for organisations that assess projects, as certification fees constitute an important source of their revenue.

Furthermore, professionals involved in the assessment of projects respond to factors related to local populations and governmental institutions [12]. Even though the increased cost of building green represents the main obstacle to sustainable construction, recognition by property owners that it is more beneficial for society is critical. The difference between developed and developing countries is important here, as the latter present a lack of public awareness about those questions, which is not necessarily the case in more western mature markets. Finally, environmental regulations suggested by experts and enforced by different national governments globally, pushed construction firms and their clients to call for specialists to help with the assessment process [19].

\section{CONCLUSION}

The analysis of NSA tools through the lens of standards and cultural specificities demonstrate they inform the planning and design of neighbourhoods adapted to a specific context by using different strategies. An NSA tool can for instance mainly use standards in order to easily export a set of fixed guidelines, to which a set of flexible criteria can eventually be added. Or on the contrary, they can be made up of parameters whose evaluation is more flexible, while making the assessment process more complex for project managers. Moreover, the selection of criteria adopted by NSA tools informs the evolution of several assessment cultures. Most of them appear to originate from a western urban planning and design tradition, which tends today to evolve according to regional actors. Those experts, pushed by government regulations, local population interests and business opportunities, go through the cultural appropriation of a given rating tool. They consequently adapt it to the cultural area in which they practice. However, we need to mention that the design of a culturally specific NSA tool does not necessarily imply that the latter is particular to the same cultural region, as demonstrated in the case of LEED-ND.

Consequently, future research needs to expand on those assumptions by analysing other NSA tools, as well as the sustainable neighbourhood projects they evaluate. Knowing that most of those tools are still designed in western developed countries, it is worth asking what will be the future trends in terms of NSA tool development. Several possibilities appear. One is that western NSA tools will probably be exported toward developing markets, therefore restraining the diversification of assessment systems and cultures. The other option is that developing countries will develop their own NSA system, therefore expanding their number and evaluation cultures. The question that comes next is: according to which different intellectual processes and strategies? Will those new tools derive directly from others such as the AQUA/HQE-A case? To which degree will they get inspired from existing tools, or present innovative approach to neighbourhood assessment? Research should then inquire 
into how those dynamics will contribute to the construction of more sustainable environments.

Beyond establishing cultural profiles, our article questions the assessment of sustainability as a practice susceptible to be exported in other countries. Indeed, the American sustainable neighbourhood model of today might contribute to the construction of the unsustainable Asian or African neighbourhoods of tomorrow. Moreover, the standards and concepts contributing to the design of a given NSA tool not only will have to be contextually adapted, but also to evolve over time. These adaptations and evolutions require the upgrade of these tools by culturally diverse experts who will have to consider practices that go beyond the ones promoted by rating systems.

\section{ACKNOWLEDGEMENTS}

This study was supported by a grant from the Palladio Foundation (France).

\section{REFERENCES}

[1] Komeily, A. \& Srinivasan, R.S., A need for balanced approach toneighborhood sustainability assessments: a critical review and analysis. Sustainable Cities and Society, 18, pp. 32-43, 2015. http://dx.doi.org/10.1016/j.scs.2015.05.004

[2] Brundtland, G.H., Report of the world commission on environment and development "Our common future", United Nations, 1987.

[3] Jabareen,Y.R., Sustainable urban forms their typologies,models, and concepts. Journal of Planning Education and Research, 26(1), pp. 38-52, 2006. http://dx.doi.org/10.1177/0739456X05285119

[4] Kyrkou, D., Taylor, M., Pelsmakers, S. \& Karthaus, R., Urban sustainability assessment systems: how appropriate are global sustainability assessment systems. Preceeding Of the 27th Conference on Passive and Low Energy Architecture, Louvain-la-Neuve Belgium, PLEA 2011.

[5] Bond, A.J., Morrison-Saunders, A. \& Howitt, R., Sustainability Assessment: Pluralism, Practice and Progress, Routledge, 2012.

[6] Sharifi, A. \& Murayama, A., A critical review of seven selected neighborhood sustainability assessment tools. Environmental Impact Assessment Review, 38, pp. 73-87, 2013. http://dx.doi.org/10.1016/j.eiar.2012.06.006

[7] Reith, A. \& Orova. M., Do green neighbourhood ratings cover sustainability? Ecological Indicators, 48, pp. 660-672, 2015. http://dx.doi.org/10.1016/j.ecolind.2014.09.005

[8] Wangel, J.,Wallhagen, M., Malmqvist, T. \& Finnveden, G., Certification systems for sustainable neighbourhoods: what do they really certify? Environmental Impact Assessment Review, 56, pp.200-213, 2016. http://dx.doi.org/10.1016/j.eiar.2015.10.003

[9] Oliveira, V. \& Pinho, P., Evaluation in urban planning: advances and prospects. Journal of Planning Literature, 24(4), pp. 343-361, 2010. http://dx.doi.org/10.1177/0885412210364589

[10] Sharifi, A., From garden city to eco-urbanism: the quest for sustainable neighborhood development. Sustainable Cities and Society, 20, pp. 1-16, 2016.

http://dx.doi.org/10.1016/j.scs.2015.09.002 
22 Urban Regeneration and Sustainability

[11] Glasson, J., Therivel, R. \& Chadwick, A., Introduction to Environmental Impact Assessment, Routledge, 2013.

[12] Sharifi, A., Sustainability at the Neighborhood Level: Assessment Tools and the Pursuit of Sustainability, Diss. Nagoya University, 2013.

[13] Berardi, U., Beyond sustainability assessment systems: upgrading topics by enlarging the scale of assessment. International Journal of Sustainable Building Technology and Urban Development, 2(4), pp. 276-282, 2011. http://dx.doi.org/10.5390/SUSB.2011.2.4.276

[14] United States Green Building Council (USGBC), Directory: project, available at http:// www.usgbc.org/projects

[15] ISO, Standards, available at http://www.iso.org/iso/home/standards.htm

[16] Giulietti, A., Harley, R.M., De Queiroz, L.P., Wanderley, M.G.L. \& Van Den Berg, C., Biodiversity and conservation of plants in Brazil. Conservation Biology,19(3), pp. 632-639, 2005.

http://dx.doi.org/10.1111/j.1523-1739.2005.00704.x

[17] Asafu-Adjaye, J., The relationship between energy consumption, energy prices and economic growth: time series evidence from asian developing countries. Energy Economics, 22(6), pp. 615-625, 2000. http://dx.doi.org/10.1016/S0140-9883(00)00050-5

[18] United States Green Building Council (USGBC), Directory: people, available at http:// www.usgbc.org/people

[19] Buckley, B. \& Logan, K.,World Green Building Trends 2016: Developing Markets Accelerate Global Green Growth, Dodge Data \& Analytics: Bedford Massachusetts, 2016. 